\title{
loffe-Regel' crossover and boson peaks in disordered solid solutions and similar anomalies in heterogeneous crystalline structures
}

Cite as: Low Temp. Phys. 34, 655 (2008); https://doi.org/10.1063/1.2967512

Published Online: 29 September 2008

I. A. Gospodarev, V. I. Grishaev, A. V. Kotlyar, K. V. Kravchenko, E. V. Manzheliĭ, E. S. Syrkin, and S. B. Feodos'ev

\section{ARTICLES YOU MAY BE INTERESTED IN}

\section{Perspective: Supercooled liquids and glasses}

The Journal of Chemical Physics 137, 080901 (2012); https://doi.org/10.1063/1.4747326

Debye to non-Debye scaling of the Boson peak dynamics: Critical behavior and local disorder in vitreous germania

The Journal of Chemical Physics 135, 174506 (2011); https://doi.org/10.1063/1.3656697

Relationship between boson heat capacity peaks and evolution of heterogeneous structure in metallic glasses

Journal of Applied Physics 115, 153505 (2014); https://doi.org/10.1063/1.4871676

LOW TEMPERATURE TECHNIQUES OPTा|CAM CAVITV PHYSICS MITIGATING THERMAL \& VIBRATIONAL NOISE

\section{DOWNLOAD THE WHITE PAPER}




\title{
loffe-Regel' crossover and boson peaks in disordered solid solutions and similar anomalies in heterogeneous crystalline structures
}

\author{
I. A. Gospodarev, ${ }^{\text {a) }}$ V. I. Grishaev, A. V. Kotlyar, K. V. Kravchenko, E. V. Manzheliĭ, \\ E. S. Syrkin, and S. B. Feodos'ev
}

B. I. Verkin Institute for Low Temperature Physics and Engineering of the National Academy of Sciences of Ukraine, pr. Lenina 47, Kharkov 61103, Ukraine

(Submitted March 11, 2008)

Fiz. Nizk. Temp. 34, 829-841 (August 2008)

\begin{abstract}
Low-frequency features of the phonon spectra of disordered solid solutions and heterogeneous crystalline structures are analyzed at the microscopic level. It is shown that boson-peak type excitations can arise in disordered solid solutions whose sites have only translational degrees of freedom. Thus it is established that such excitations appear mainly because of the additional positional dispersion of sound waves which is due to the disordering. The influence of boson-peak excitations on the low-temperature specific heat is investigated. It is found that in a number of cases the specific heat is more sensitive to excitations of this kind than the low-frequency density of states is. It is shown that anomalies similar to Ioffe-Regel' crossover and boson peaks can also arise in disordered heterogeneous crystalline structures with a complicated lattice.
\end{abstract}

(C) 2008 American Institute of Physics. [DOI: 10.1063/1.2967512]

\section{INTRODUCTION}

Various disruptions of the crystalline regularity in the arrangement of atoms make a substantial change in phonon and other quasiparticle spectra as well as the thermodynamic and kinetic characteristics due to them. I. M. Lifshits and his school laid the foundation for the systematic theoretical study of the properties of real crystals and disordered systems. ${ }^{1-4}$ Many features of the dynamics of a crystal lattice containing defects have now been studied in detail and are described in monographs and textbooks, among which A. M. Kosevich's books stand out. ${ }^{5-7}$

Even though substantial advances have been made in the study of systems with different types of disorder and general approaches and methods for describing such systems theoretically have been developed, ${ }^{4}$ the study of the characteristics of their phonon spectra (specifically, at low frequencies) and the associated low-temperature thermodynamic characteristics remains an exceptionally important and topical problem of modern solid-state physics. In recent years more attention has been directed toward studying the properties of and the conditions under which a so-called "boson peak," characteristic of the phonon spectra of such systems, arises. Initially, it was observed in experiments on inelastic neutron or light (Mandel'shtam-Raman) scattering as a maximum in the frequency dependence of the ratio of the density of vibrational states $g(\omega)$ (or the scattering intensity $I(\omega) \sim g(\omega)$ ) to the squared frequency $\omega^{2}$. This maximum appears in the low-frequency region of the vibrational density of states at frequencies between 0.5 and $2 \mathrm{THz}{ }^{8}$ i.e. substantially below the Debye frequency. A peak does not arise in the function $g(\omega)$ itself. $^{9}$

Boson peaks were subsequently also investigated in experiments on inelastic scattering of different particles. ${ }^{10-15}$ All this has stimulated the appearance of a large number of theoretical works on determining the nature of a boson peak. Analytical methods, based on various assumptions about the appearance of disorder, ${ }^{16-29}$ as well as computer simulation $^{30-36}$ have been used to study the interaction of local defects, which give rise to disorder, with sound waves. Some investigators attribute the appearance of a boson peak to the appearance of quasilocal vibrations in such a system. Models with a random distribution of harmonic force constants, which also lead (in the opinion of the authors) to the appearance of boson-peak anomalies, have also been examined. The position of a boson peak corresponds to the frequency at which the disorder parameter, which is understood to be the distance at which acoustic phonons propagate with the speed of sound, ${ }^{37}$ becomes comparable to the wavelength (Ioffe-Regel' limit ${ }^{38}$ ). In Ref. 37 Ioffe-Regel' crossover and the appearance of a boson peak were interpreted by means of numerical simulation as a transition from so-called "propagons" (rapidly propagating low-frequency phonons with an acoustic dispersion law) to "diffusons"-phonons whose propagation is retarded by scattering by localized states which arise at higher frequencies.

We call attention especially to A. M. Kosevich's contribution to the study of the relation between the Ioffe-Regel' effect and the appearance of a boson peak. ${ }^{39,40}$ In these works it is shown that the Ioffe-Regel' limit is related with effects based on the interaction of rapidly propagating quasiparticles (of the acoustic-phonon type) with "slower" quasiparticles. Such Ioffe-Regel' crossover results in the appearance of features similar in form to the first van Hove singularity, which, as is well known, ${ }^{5-7}$ is determined by the dimension of the lattice, in the phonon density in systems with crystal order.

The so-called phonon-fractal model ${ }^{42}$ and the log-normal frequency distribution model ${ }^{43}$ were used in Ref. 41 to in- 

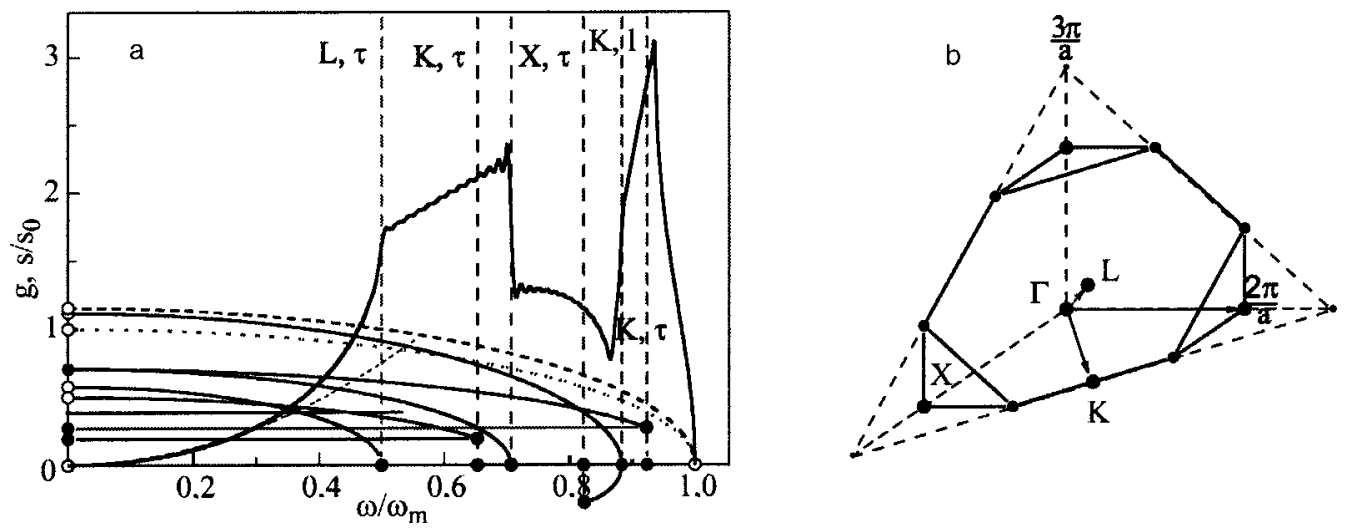

FIG. 1. The phonon density of states (thick solid line) and frequency dependences of the group velocities of phonon modes (thin lines) along the principal high-symmetry crystallographic directions of a fcc crystal with central interaction of the nearest neighbors (solid lines-along the direction $\Gamma \mathrm{K}$; dashed lines-along the direction $\Gamma \mathrm{L}$; dot-dashed lines-along $\Gamma \mathrm{X}$ (a); first octant of the first Brillouin zone of a fcc crystal lattice $s_{0}=\left(a \omega_{m}\right) / 4(\mathrm{~b}), a-l a t t i c e$ constant.

vestigate the anomalies in disordered systems.

However, even though there are plenty of theoretical models describing systems whose spectra contain a boson peak and experimental results on observing such a peak, there is still no firm opinion about the nature of this peak. For example, in Ref. 44, whose objective is to observe a boson peak on the surface of porous silicon experimentally, it is stated directly that the nature of the boson peak remains unclear in spite of the many efforts made. Essentially the same result is obtained in Ref. 45, where the relation between the boson peak and the first van Hove singularity is underscored. We also note that a possible manifestation of a boson peak was experimentally observed in investigations of the isochoric thermal conductivity of molecular cryocrystals. $^{46}$

The present work is devoted to explaining on a microscopic level the nature of Ioffe-Regel' type anomalies and the boson peak in the low-frequency range of the phonon spectrum of disordered systems which consist of particles possessing only translational degrees of freedom. It is demonstrated that similar anomalies even arise in ordered heterogeneous crystalline structures, for example, strongly anisotropic crystals with a complicated lattice. It is also shown that the study of the thermodynamic characteristics, such as the low-temperature specific heat and the temperature dependence of the Debye temperature, can often be even more informative than the direct study of the density of states.

\section{TEMPERATURE DEPENDENCE OF THE DEBYE TEMPERATURE AS A CHARACTERISTIC OF THE PROPAGATION OF ACOUSTIC PHONONS}

It is well-known that in the Debye approximation the real vibrational spectrum of a crystal is replaced with phonons having an acoustic dispersion law $\omega=s k$, where $s$ is the speed of sound and $k$ is the modulus of the wave vector. The corresponding density of states has the form (see, for example, Refs. 5-7)

$$
g_{D}^{(q)}(\omega)=\frac{q \omega^{q-1}}{\omega_{D}^{q}},
$$

where $q$ is the dimension of the lattice. As $k$ increases, the phonon dispersion relation in a crystal increasingly deviates from a linear law (the frequency $\omega$ becomes less than $s k$ ) and the real density of states moves upward from the relation (1). Finally, for some value of the modulus $k^{*}$ of the wave vector the group velocity of some transverse acoustic phonon modes vanishes in one of the crystallographic directions. As a rule, this direction is also the direction of one of the symmetry axes in $\mathbf{k}$ space, and the value $k^{*}$ corresponds to the boundary of the first Brillouin zone in this direction. Thus, a transition from closed to open isofrequency surfaces occurs at the frequency $\omega^{*}=\omega\left(k^{*}\right)$ and the value of $\omega^{*}$ is the frequency of the first van Hove singularity.

Figure 1 displays the density of states of an fcc crystal lattice with a central interaction between nearest neighbors and the frequency dependence of group velocities of longitudinal and transverse phonons along the high-symmetry crystallographic directions $\Gamma \mathrm{X}, \Gamma \mathrm{L}$, and $\Gamma \mathrm{K}$ (for convenience, the first octet of the first Brillouin zone of an fcc crystal is shown on the right-hand side).

It is evident that the Debye density of states $g_{D}^{(3)}(\omega)$, given by the relation (1) with $q=3$ and shown by a thin dashed line in the figure, agrees quite satisfactorily with the true density of states for $\omega \leqslant 0.25 \omega_{m}\left(\omega_{m}\right.$ is the upper limit of the band in the quasicontinuous spectrum). For $\omega>0.25 \omega_{m}$ the curve $g(\omega)$ starts to deviates upwards from the curve $g_{D}^{(3)}(\omega)$. The van Hove singularity corresponds to vanishing of the group velocity of transverse phonons propagating in the direction $\Gamma \mathrm{L}$. For this model the frequency $\omega^{*}=\omega_{m} / 2$. The character of the phonon dispersion becomes qualitatively different for $\omega \geqslant \omega^{*}$. Together with rapidly propagating phonons, whose dispersion law is still close to linear (as the figure shows, the function $s(\omega)$ for the other branches and directions of propagation is comparatively small for $\left.\omega \approx \omega^{*}\right)$, localized states arise- transverse phonons no longer propagate in the direction $\Gamma \mathrm{L}$. Similarly, at the frequency $\omega^{* *}$ $=\omega_{m} / \sqrt{2}$ (the Einstein frequency for the present model) transverse phonons no longer propagate in the direction $\Gamma \mathrm{X}$. The density of states $g(\omega)$ in the interval $\left[\omega^{*}, \omega^{* *}\right]$ has the linear form characteristic for the Debye density of states of a two-dimensional crystal $g_{D}^{(2)}(\omega)$. This indicates that the propagation of long-wavelength phonons at these frequencies is of a two-dimensional character. 

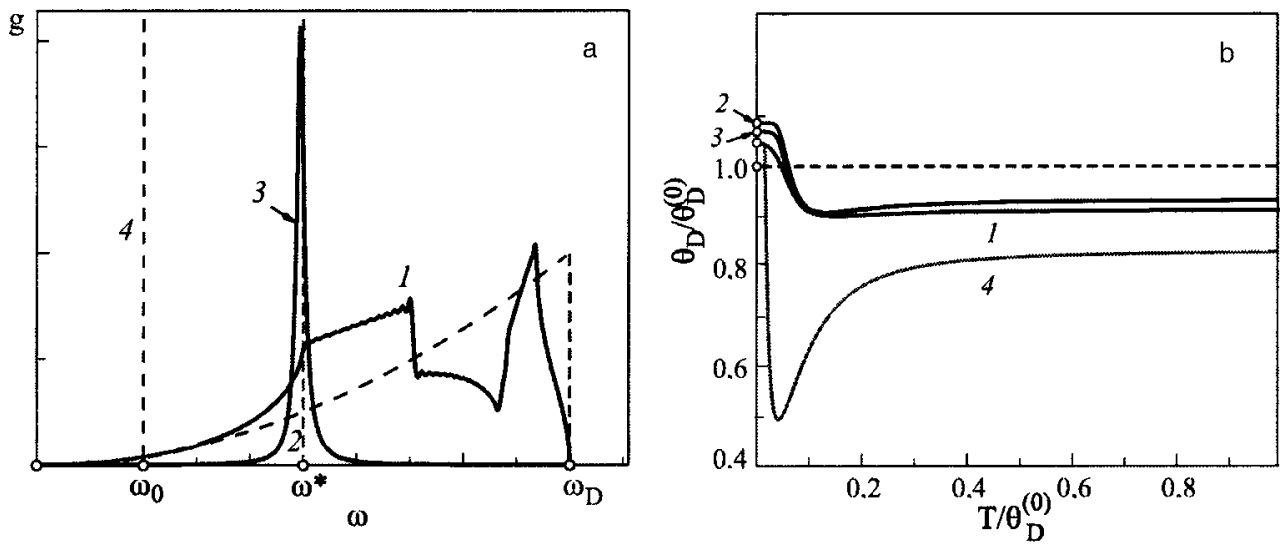

FIG. 2. Relation between the temperature dependence of the Debye temperature and the character of the propagation of long-wavelength phonons in a crystal. The curves 1 (a) and (b) correspond to a fcc crystal with central interaction of the nearest neighbors; curves 2-4-superposition of the Debye density of states with localized and quasilocalized modes $2-4$, respectively.

Of course, when the phonon density deviates from the form (1) the temperature dependence of the phonon specific heat deviates from its Debye form $C_{D}(T)$ (see, for example, Refs. 5-7). In addition, this deviation will be all the stronger the lower the frequencies at which such deviation starts. Ordinarily, the deviation of the true phonon specific heat from $C_{D}(T)$ is described as a temperature dependence of the Debye temperature $\Theta_{D}$. This dependence can be found from the transcendental equation

$$
\begin{aligned}
& C_{v}(T)=C_{D}(T) \equiv 3 R\left\{D\left(\frac{\Theta_{D}}{T}\right)-\frac{\Theta_{D}}{T} D^{\prime}\left(\frac{\Theta_{D}}{T}\right)\right\} ; \\
& D(x) \equiv \frac{3}{x^{3}} \int_{0}^{x} \frac{z^{3} d z}{e^{z}-1}
\end{aligned}
$$

where the specific heat is determined experimentally or from a microscopic calculation using the relation

$$
C_{v}(T)=3 R \int_{0}^{\omega_{m}}\left(\frac{\hbar \omega}{2 k T}\right)^{2} \operatorname{sh}^{-2}\left(\frac{\hbar \omega}{2 k T}\right) g(\omega) d \omega
$$

(of course, for $g(\omega)=g_{D}^{(3)}(D)$ the expressions (2) and (3) are identical, and $\left.\omega_{D}=\omega_{m}\right)$.

Thus, the temperature dependence $\Theta_{D}(T)$, presented as the curve $l$ in Fig. $2 b$, corresponds to a fcc crystal with central interaction between nearest-neighbors (Fig. 2a, curve 1). The strong temperature dependence of $\Theta_{D}$ for $T \ll \Theta_{D}$ invites our attention. This is characteristic for a very large number of compounds (see, for example, Ref. 47). At the same time, at low temperatures long-wavelength phonons with an acoustic dispersion law make the main contribution to the specific heat. At first glance, the relation (2) should describe the temperature dependence $C_{v}(T)$ well at low temperatures and the Debye temperature should differ little from the value $\Theta_{D}^{(0)} \equiv \hbar \omega_{D} / k$.

To determine the reason for the strong temperature dependence of $\Theta_{D}$ for $T \ll \Theta_{D}$, we shall examine the function $\Theta_{D}(T)$ for a system whose phonon density of states is a linear combination of the function $g_{D}^{(3)}(\omega)$ (dashed line in Fig. 2a) and the Einstein density of states $\delta\left(\omega-\omega^{*}\right)$ for the frequency of the first van Hove singularity (line 2 on the same fragment). The function obtained $\Theta_{D}(T)$ is represented as the curve 2 in Fig. 2b. The fact that it matches completely satisfactorily with the curve $\Theta_{D}(T)$ for a fcc crystal, specifically, the rapid drop-off for $T \leqslant 0.1 \Theta_{D}^{(0)}$ and especially the matching of the minima (with respect to temperature and magnitude), shows that the function $\Theta_{D}(T)$ can be explained by the change in the character of phonon propagation at the frequency of the first van Hove singularity-the appearance of localized states together with rapidly propagating acoustic phonons. Taking account of the smearing of the Einstein level, as one can see in Fig. 2 (curves $3 a$ and $3 b$ ), somewhat improves the fit of the function $\Theta_{D}(T)$ at low temperatures.

For $\omega<\omega^{*}$ the average group velocity of the phonons decreases smoothly as the frequency increases, and at $\omega$ $=\omega^{*}$ it abruptly decreases further. Thus, the frequency of the first van Hove singularity in an ideally ordered crystal is actually a boundary between fast and slow phonons ("propagons" and "diffusons," in terms of Ref. 37), and it can be regarded as the analog of Ioffe-Regel' crossover in a regular crystalline system.

It makes sense to examine boson peaks as maxima of the ratio $g(\omega) / \omega^{2}$ only for $\omega<\omega^{*}$, since a maximum corresponding to the first van Hove singularity is always present in this ratio. The phonon density in this frequency interval ("propagon band") can be approximated by a parabola, and its deviation from the Debye density $g_{D}^{(3)}(\omega)$ can be expressed in terms of the frequency dependence of $\omega_{D}$, i.e. the phonon density can be written in a form similar to the expressions (1). For $q=3$ we have

$$
g(\omega) \equiv \frac{3 \omega^{2}}{\omega_{D}^{3}(\omega)} .
$$

Then, using the definition of $\omega_{D}$ (see, for example, Ref. 48), the ratio of the phonon density to the squared frequency can be expressed in terms of the dispersion of the sound speeds $s_{i}(\omega)$

$$
\frac{g(\omega)}{\omega^{2}} \equiv \frac{3}{\omega_{D}^{3}(\omega)}=\frac{V_{0}}{6 \pi^{2}} \sum_{i=1}^{3} \frac{1}{s_{i}^{3}(\omega)} .
$$

where $V_{0}$ is the volume of a unit cell. Thus the appearance of a maximum in the ratio $g(\omega) / \omega^{2}$ is due to the additional dispersion of the sound speeds, which could be due to struc- 

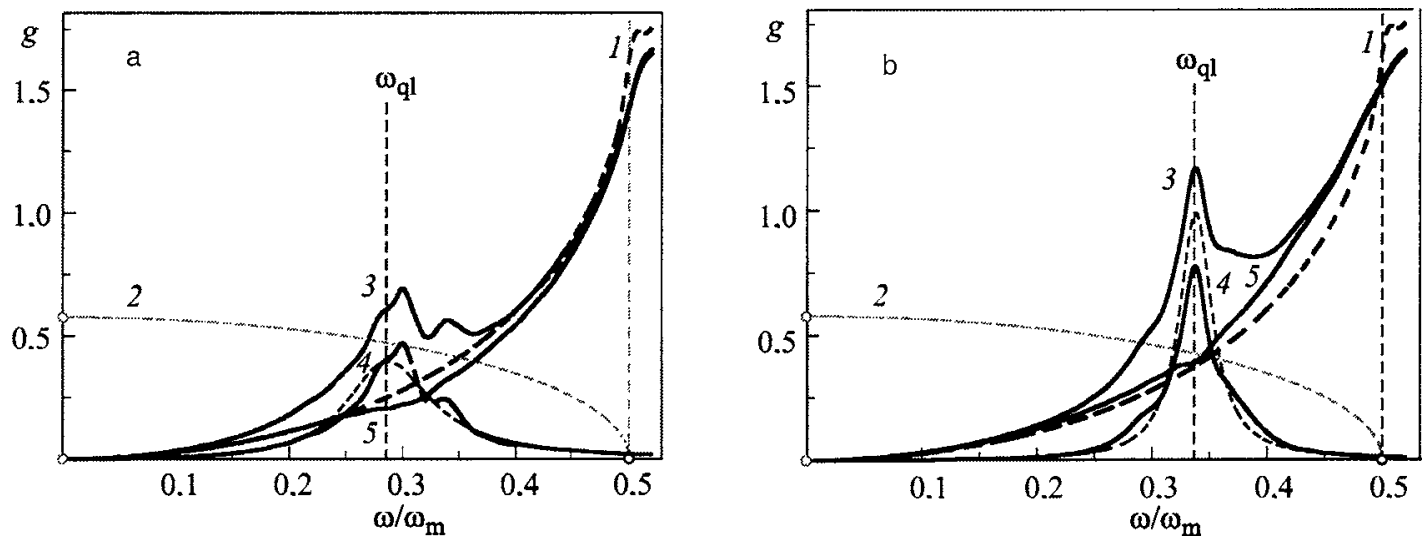

FIG. 3. Low-frequency parts of the spectra of 5\% solid solutions of a heavy isotopic impurity (a) and impurity with a weakened force interaction (b). The phonon densities of an ideal lattice (1); frequency dependence of the transverse sound velocity in the $\Gamma L$ direction (2); phonon densities of the solution (3); contributions of impurity atoms and crystal-matrix atoms to these densities (4) and (5).

tural nonuniformity as a source of quasilocalized vibrations-defects, complicated unit cell structure, rotational degrees of freedom of the crystal lattice sites, and so forth.

Such additional dispersion of the sound speeds should be manifested in the behavior of the temperature dependence $\Theta_{D}(T)$. If the additional "retardation" of the sound waves forms a maximum in the frequency dependence of the ratio $g(\omega) / \omega^{2}$, then a low-temperature minimum should arise in the curve of $\Theta_{D}(T)$, and this minimum should be deeper than in the curves 1-3 presented in Fig. 2b. Such a minimum occurs in the curve 4 in Fig. 2 b. This curve is the temperature dependence of the Debye temperature, corresponding to the presence of a resonance level with frequency $\omega_{0}=\omega_{D} / 5$ (line 4 in the same fragment), in addition to the quasilocalized excitation represented by the curve 3 in Fig. $2 a$, in the phonon spectrum $g_{D}^{(3)}(\omega)$. A minimum can also appear in the function $\Theta_{D}(T)$ when a maximum is not observed in the ratio $g(\omega) / \omega^{2}$, since it does not fall into the "propagon" region.

In the subsequent sections of this article we examined the manifestations of boson peaks and Ioffe-Regel' crossover in the phonon spectra of solid solutions and some other heterogeneous structures, both disordered and possessing crystalline regularity in the arrangement of the atoms. It is demonstrated that they are related with the additional dispersion of the long-wavelength acoustic phonons and that this additional dispersion affects the temperature dependence of the Debye temperature.

\section{INTERACTION OF ACOUSTIC PHONONS WITH QUASILOCALIZED STATES}

It is well-known (see, for example, Refs. 5-7) that heavy or weakly bound impurities can form in the low frequency part of a band in the quasicontinuous spectrum (its "propagon" part) maxima in the phonon density of statesthe so-called quasilocal vibrations. Such vibrations propagate with very low velocities, and their propagation can be represented in the form of spherical waves

$$
\rho(\omega, \mathbf{r})=\frac{1}{\pi} \operatorname{Im} G(\omega, \mathbf{r})
$$

$(G(\omega, \mathbf{r})$ is a Green's function corresponding to the displacement of an atom with the radius vector $\mathbf{r}$ ), which qualitatively differ from the spectral densities of the atoms of the main lattice, including atoms with impurity neighbors. They have a quasiresonance form, and features such as van Hove singularities are in no way manifested in them. Thus, Fig. 3 shows the low-frequency $\left(\omega \leqslant \omega_{m} / 2\right)$ regions of the phonon spectra of solid solutions of a heavy isotopic impurity $\mathrm{m}^{\prime} / \mathrm{m}=4$ (a) and an impurity which is bound four times more weakly with the main lattice than the atoms of the main lattice are bound with one another (b). Both fragments of the figure show the phonon density of an ideal crystal (fcc crystal lattice with central interaction between nearestneighbors-dashed curves 1), and the frequency dependence of the transverse velocity of sound in the direction $\Gamma \mathrm{L}$, whose vanishing determines the position of the first van Hove singularity (curves 2). The curves 1 and 2 (same designations) are also presented in Fig. 4 and 5.

The impurity concentration is $p=5 \%$ in both cases. It is evident that low-frequency peaks-quasilocal vibrationsform in the phonon densities (curves 3). For a weakly bound impurity a quasilocal peak has a distinct resonance character. The curves 4 in both fragments correspond to the contribution of impurity atoms $g_{\text {imp }}(\omega)$ to the phonon density. These curves deviate negligibly from the frequency dependences (shown next to them as dashed lines) multiplied by the concentration of the spectral density of an isolated impurity atom $p \rho(\omega, \mathbf{r})$. The function $g_{\text {imp }}(\omega)$ differs from zero only near the maximum in the curve $\rho(\omega, \mathbf{r})$-quasilocal frequency $\omega_{q l}$. Consequently, the quasilocal vibrations can be represented as spherical waves moving slowly away from an impurity.

The curves 5 in both fragments of the figure represent the functions $g(\omega)-g_{\text {imp }}(\omega)$, i.e. the contributions of the vibrations of atoms of the main lattice to the phonon density of the solution. At frequencies $\omega<\omega_{q l}$ these vibrations propagate in the form of plane waves and the corresponding sections in the curves 5 are smooth and have a parabolic (quasi- 

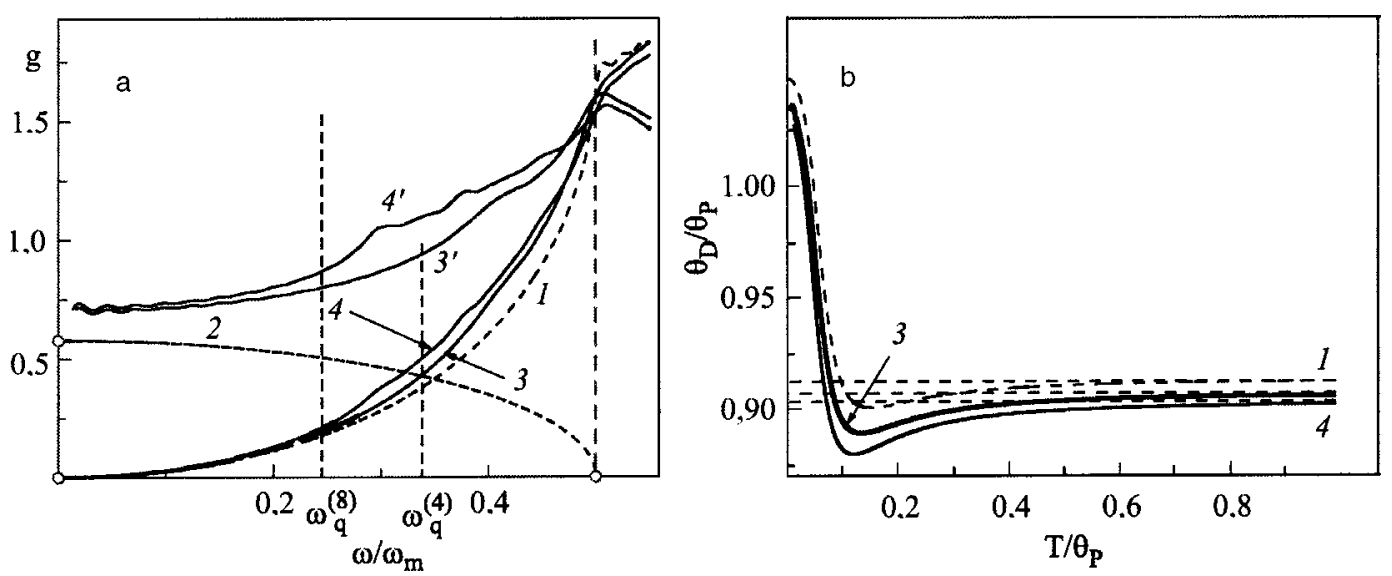

FIG. 4. Low-frequency parts of the phonon spectra (a) and temperature dependences of $\Theta_{D}$ (b) for fcc crystals $5 \%$ of whose force bonds are weakened. The dashed lines 1 and 2 correspond to the phonon density and frequency dependence of the sound velocity in the direction $\Gamma L$ for an ideal crystal. Line 3-phonon densities (a) and $\Theta_{D}(T)$ (b) for the case of bonds weakened by a factor of 4; line 4-weakening by a factor of 8. Lines 3 ' and 4' - ratios of the density of states to the squared frequency $\Theta_{p}=\hbar \omega_{m} / k$.

Debye) form. At frequencies close to $\omega_{q l}$ the plane waves are scattered by waves moving slowly away from the impurities. A kink, similar to the kink at the frequency $\omega^{*}$ of the first van Hove singularity, forms in the frequency dependence $g(\omega)-g_{\text {imp }}(\omega)$. Both features have similar natures-this is scattering of fast acoustic phonons by slow phonons.

Thus, the effect of impurity atoms which are heavy or weakly bound with the atoms of the main lattice on the phonon spectrum and the vibrational characteristics reduces to the formation of quasilocal vibrations (curves 4), which are due to the impurity of vibrations themselves, and to scattering by these vibrations of fast acoustic phonons formed by the vibrations of the atoms of the main lattice. The kinks in the curves 5 are actually manifestations of Ioffe-Regel' crossover, but in this case these features are "hidden" under the quasilocal peaks.

Such features can be observed in systems where the weakening of the interatomic interaction is not accompanied by the formation of quasilocal modes or in systems where the frequencies of the quasilocal vibrations of impurity atoms lie above $\omega^{*}$. Thus, Fig. 4 displays the low-frequency parts of the phonon densities of states (a) and the temperature dependences of $\Theta_{D}$ (b) for a fcc crystal lattice, where $5 \%$ of the force bonds are weakened by factors of 4 and 8 (the curves 3 and 4 in both fragments, respectively). Figure $4 \mathrm{a}$ also displays the functions $(g(\omega) / 4)\left(\omega_{m} / \omega\right)^{2}$ (the curves $3^{\prime}$ and $4^{\prime}$, respectively) in which the deviation of the phonon density of states from the Debye form is more distinct. It is evident that the phonon densities start to differ qualitatively from $g_{D}^{(3)}(\omega)$ at the frequencies $\omega=\omega_{q l}^{(8)}$ (curve 3) and $\omega$ $=\omega_{q l}^{(8)}$ (curve 4), i.e. at quasilocal frequencies corresponding to the presence of an isolated weakly bound impurity in the lattice $\left(\alpha^{\prime} / \alpha=1 / 4\right.$ and $\alpha^{\prime} / \alpha=1 / 8$, respectively). The excitation of slowly propagating waves with such a frequency attests to the presence of atoms in which several bonds are weakened (at least, two along the same straight line), i.e. the formation of defect clusters (or impurity molecules), in the system. An example of such a defect cluster is an isolated substitution impurity with altered force bonds (in this case the nearest neighbors of the impurity are also defect atoms). The minimum size of such a defect cluster equals two interatomic distances.

The deviation of the phonon density of states from $g_{D}^{(3)}$ $\times(\omega)$ at low frequencies should noticeably affect the behavior of the low-temperature thermodynamic characteristics. Figure $4 \mathrm{~b}$ shows clearly that appreciable low-temperature minima are present in the curves 3 and 4 , which represent
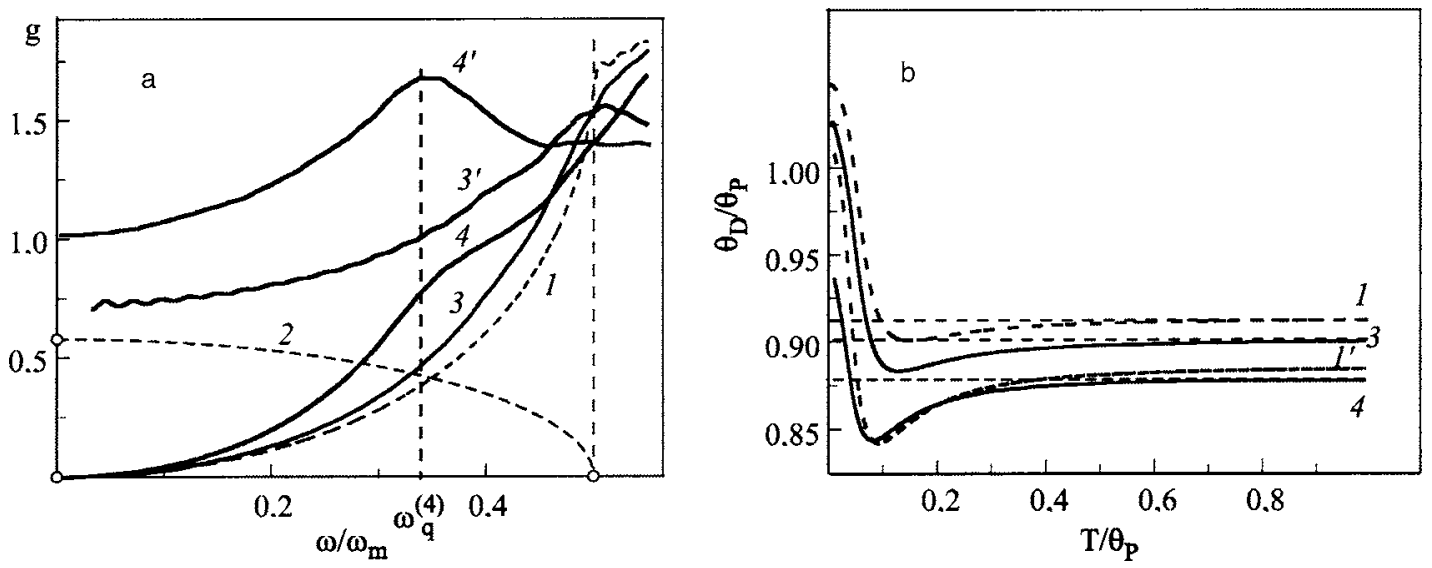

FIG. 5. Low-frequency parts of the phonon spectra (a) and temperature dependences $\Theta_{D}(T)$ (b) of solid solutions of a light weakly bound impurity in a fcc crystal. The notation is the same as Fig. 4 with $p, \%: 5(3)$ and $\left(3^{\prime}\right), 25(4)$ and $\left(4^{\prime}\right)$. The curve $1^{\prime}$ (b) corresponds to a 5\% solution of weakly bound impurities. 

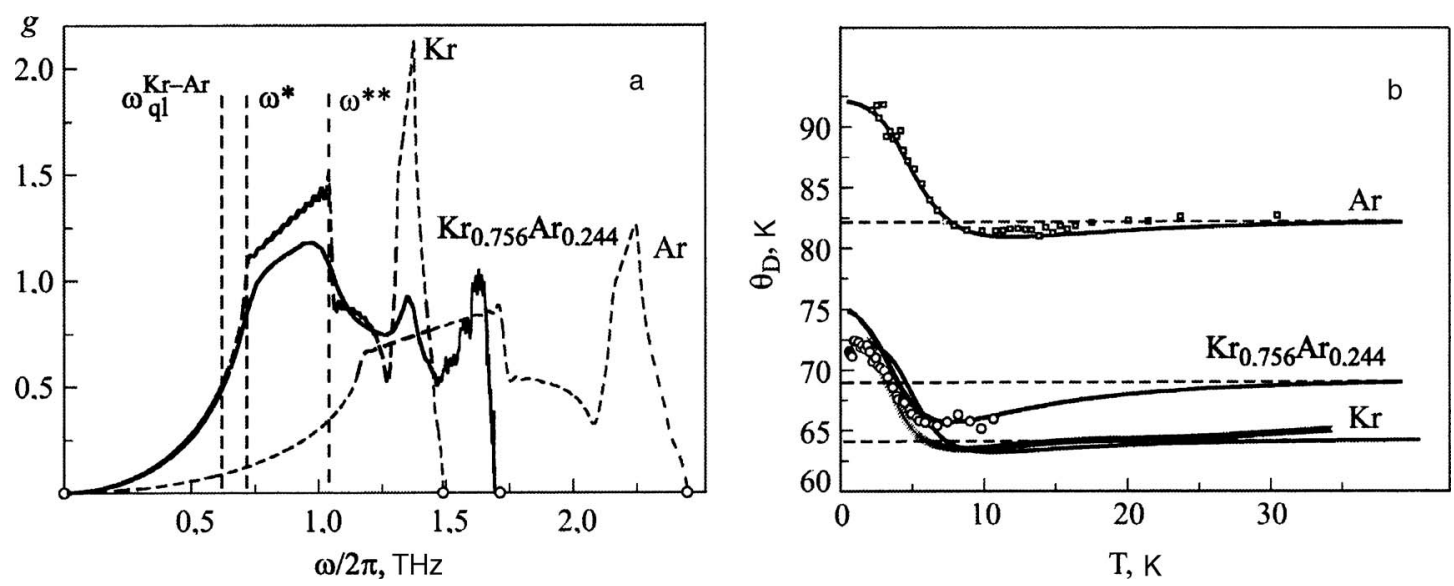

FIG. 6. The phonon densities (a) and temperature dependences of the Debye temperature (b) for krypton, argon, and the solid solution $\mathrm{Kr}_{0.756} \mathrm{Ar}_{0.244}$. Solid lines - computational result, points-calculation based on experimental measurements of the specific heat ${ }^{49,50}$ (b).

$\Theta_{D}(T)$ for crystals with force bonds weakened by factors of 4 and 8 , respectively; these minima indicate retardation of acoustic phonons as a result of their localization on weak bonds and as result of additional scattering of phonons which have remained delocalized on the quasilocalized states formed.

Figure 5 shows similar curves for 5\% (curves 3 and $3^{\prime}$ ) and $25 \%$ (curves 4 and $4^{\prime}$ ) solid solutions of a light weakly bound impurity $\left(m^{\prime} / m=\alpha^{\prime} / \alpha=1 / 4\right)$. The curve $4^{\prime}$ contains a distinct maximum, which can be interpreted as a boson peak. In addition, its frequency equals the quasilocal frequency $\omega_{q l}^{(4)}$, excited in the crystal by an isolated substitution impurity bound with surrounding atoms four times more weakly than the atoms of the main lattice. In the present system, such vibrations are characteristic for main-lattice atoms whose neighbors are several light weakly bound impurities, i.e. atoms which have become weakly bound defects on account of being neighbors of impurities. For a $25 \%$ concentration of light weakly bound impurities the number of such defect atoms is already quite large; at $5 \%$ the probability of their formation is still small.

Curve 3 in Fig. 5b, which displays the corresponding curves $\Theta_{D}(T)$, exhibits a quite appreciable minimum, which indicates that the low-temperature specific heat is highly sensitive to the retardation of long-wave length acoustic phonons. The temperature and magnitude of the minimum in the curve 4 are identical to these quantities in the curve 1 , representing the function $\Theta_{D}(T)$ for a $5 \%$ solution of weakly bound impurities. Therefore, $\sim 5 \%$ weakly bound defects are formed in a $25 \%$ solution of light weakly bound impurities.

The high sensitivity of the low-temperature specific heat to retardation of long-wavelength acoustic phonons is clearly manifested when it is not so much the interaction of impurities with main-lattice atoms that is weakened as is the interaction between substitution impurities in the matrix of the main lattice. An example of such a system is the solid solution $\mathrm{Kr}_{1-p} \mathrm{Ar}_{p}$. Krypton and argon dissolved well in one another, and the concentration $p$ can assume any value from 0 to 1 . Argon is $\approx 2.09$ times lighter than krypton, and the interaction of argon impurities with krypton atoms (for low Ar concentrations) is slightly weaker than the interaction of krypton atoms with one another, i.e. an isolated Ar impurity in a $\mathrm{Kr}$ matrix essentially behaves like a light isotope. At the same time the interaction of argon impurities in a krypton matrix with one another is more than five times weaker than the interaction between krypton atoms. ${ }^{49}$

Figure 6 displays the phonon densities of pure krypton and argon (dashed lines) and the solid solution $\mathrm{Kr}_{0.756} \mathrm{Ar}_{0.244}$ as well as the function $\Theta_{D}(T)$ for these systems. At this concentration the phonon spectrum of the solution is formed, on the one hand, by an increase in the number of high-frequency states $^{50}$ as a result of isolated impurities as well as defect clusters less than two interatomic distances in size. Weakly bound argon impurities do not form in such clusters and quasilocal vibrations due to them also do not form. On the other hand, for $p \approx 25 \%$ (as shown in the preceding case) quite a large number of larger defect clusters form and they form similar quasilocal vibrations. At the same time (in contrast to the preceding case) the frequency of these quasilocal vibrations $\omega_{q l}^{\mathrm{Kr}-\mathrm{Ar}}$ is only somewhat lower than the frequency of the first van Hove singularity of krypton $\omega^{*}\left(\omega_{q l}^{\mathrm{Kr}-\mathrm{Ar}}\right.$ $\left.\approx 0.86 \omega^{*}\right)$. Consequently, no singularities appear in the phonon density of the solution or in the ratio of the phonon density to the squared frequency.

As is evident in Fig. 6, the broadening of the band in the quasicontinuous spectrum, as compared with krypton, occurs in a solution mainly as a result of phonons with frequencies in the interval $\left[\omega^{*}, \omega^{* *}\right]$ (i.e. "diffusons"). On most of the frequency interval $\left[0, \omega^{*}\right]$ the phonon densities of the solution and pure krypton are virtually the same. Such a redistribution of phonon frequencies results in a characteristic double-extremum behavior of the temperature dependence of the relative change of the low-temperature specific heat, ${ }^{49}$ the maximum in which attests to the additional retardation of long-wavelength acoustic phonons by slow phonons, corresponding to quasilocal vibrations of weakly bound argon atoms. As in the previously considered cases, this gives rise to the formation of an appreciable minimum in the temperature dependence of $\Theta_{D}$. Figure $6 \mathrm{~b}$ shows the functions $\Theta_{D}(T)$ for pure krypton and argon as well as the solution $\mathrm{Kr}_{0.756} \mathrm{Ar}_{0.244}$. The temperature dependences of the Debye temperature are reconstructed for values of the specific heat calculated theoretically (solid lines) as well as determined experimentally (points). ${ }^{49}$ Evidently, the theoretical results demonstrate good 
agreement with experiment, specifically, near the minimum in the function $\Theta_{D}(T)$ for the solution.

The results presented in the present section show that the low-temperature specific heat and, correspondingly, the temperature dependence of $\Theta_{D}$ are highly sensitive not only to the formation of quasilocal states but also to a decrease of the propagation velocity of long-wavelength acoustic phonons as a result of their scattering by these states. In order that the results of such retardation be clearly manifested in the form of boson peaks in the ratio $g(\omega) / \omega^{2}$ or additional singularities of the Ioffe-Regel' type in the frequency interval $\left[0, \omega^{*}\right]$ for defects consisting of a local weakening of interatomic interactions or light weakly bound to impurities, the following conditions must be satisfied. First, the frequency of the scattering quasilocal vibrations must be sufficiently low, i.e. the defect must be sufficiently "strong." Second, a defect cluster must be quite large (at least two interatomic distances), which requires a sufficiently high defect concentration $\sim 15-20 \%$. The second condition signifies the appearance of another parameter with the dimension of length $l \sim 2 \pi s_{0} / \omega>a_{0},{ }^{40}$ where $a_{0}$ is the interatomic distance, in the system. In our case this parameter plays the role of a disorder parameter.

\section{LOW-FREQUENCY FEATURES OF THE PHONON SPECTRA OF LAYERED CRYSTALS WITH A COMPLEX LATTICE}

It was shown in the preceding section that the lowfrequency features of the phonon spectra, which distinguish the spectra from the Debye form, are due to the scattering of fast long-wavelength acoustic phonons by quasilocalized vibrations, waves slowly diverging away from a site. The sources of such quasilocalized vibrations are various structural nonuniformities whose characteristic size should be, at least, several interatomic distances. Generally speaking, it is not at all necessary that these nonuniformities destroy the crystalline regularity in the arrangement of the atoms (i.e. they need not be defects). There exist in nature an enormous number of compounds with complex crystal lattices whose unit cells contain a large number of atoms. If the force interaction between some atoms of a single unit cell is much weaker than the interaction between cells, optical branches with frequencies much lower than the Debye frequency of the given compound arise in the phonon spectrum of the crystal. These optical branches can intersect the acoustic branches, giving rise to additional features in the phonon density of states at frequencies less than the frequency of the first van Hove singularity.

The presence of low-lying optical modes in the spectrum is characteristic of many strongly anisotropic layered crystals (see, for example, Refs. 51-53). We note at the outset that the deviation of the phonon spectrum of such compounds at low frequencies can also be due to a manifestation of their quasi-low-dimensional structure ${ }^{54}$ as well as the possible effect of flexural stiffness of individual layers. ${ }^{55}$ However, the crossing of low-lying optical modes with acoustic modes is characteristic of a large number of layered structures, including structures where neither quasi-low-dimensional features nor flexural vibrations are manifested in the low-frequency region of the phonon spectrum. These structures include high-temperature superconductors, transition-metal dichalcogenides, structures based on cobalt dioxide, a number of polymers and biopolymers, as well as many other natural and synthesized materials.

A distinguishing feature of the structure of such compounds is alternation of layers with strong interatomic interaction (covalent or metallic) with layers where the interatomic interaction is much weaker, for example, van der Waals interaction. Since this interaction is weak in all directions, the propagation of long-wavelength phonons is of a three-dimensional character and can be characterized, specifically, by the temperature dependence of $\Theta_{D}$, determined using the relations (2) and (3).

We shall examine a model system based on a fcc crystal lattice by "separating" the lattice along the [111] axis into a structure of the type $\ldots-B-B-A-B-B-A-B-B-\ldots$ In this case the $A$ and $B$ atoms form close-packed layers. To describe the interatomic interaction, we shall confine our attention, once again, to a central interaction between nearestneighbors. We shall assume that the interaction between type $B$ atoms (lying in one layer as well as in different layers) is half the interaction between type $A$ atoms and atoms of different types (we shall assume these interactions to be the same).

The phonon spectrum of this model contains nine branches (three acoustic and six optical), and the optical modes are not separated from the acoustic modes by a gap. The frequencies of all phonons polarized along the [111] axis (in what follows we shall designate this axis as the $c$ axis, the

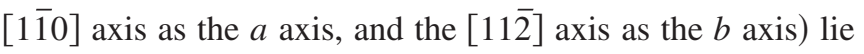
in the low-frequency region. At $k=0$ two optical modes have low frequencies, which correspond to a change in the topology of the isofrequency surfaces (from closed to open along the $c$ axis) for transverse and longitudinal modes, respectively. Thus, these frequencies play the role of van Hove frequencies $\omega^{*}$. These frequencies are designated in Figs. 7 and 8 by the vertical dashed lines $\omega_{\tau}^{*}$ and $\omega_{l}^{*}$.

Figure 7 displays the spectral densities (6) corresponding to displacements of $A$ and $B$ atoms along the $c$ axis and in the basal plane $a b$ (curves 1 ). The normalization of each spectral density corresponds to its contribution to the total phonon density of states $g(\omega)$ presented in Fig. 8a:

$$
g(\omega)=\frac{2}{9} \rho_{a b}^{(A)}(\omega)+\frac{1}{9} \rho_{c}^{(A)}(\omega)+\frac{4}{9} \rho_{a b}^{(B)}(\omega)+\frac{2}{9} \rho_{c}^{(B)}(\omega)
$$

Figure 7 also displays the quantities proportional to the ratio of the corresponding spectral densities to the squared frequency (curves 2). The coefficients of proportionality are chosen from considerations of convenience in locating these curves in the same coordinate grid as the corresponding spectral density.

The functions $\rho_{c}^{(A)}(\omega)$ and $\rho_{c}^{(B)}(\omega)$ (and their ratios to $\omega^{2}$ ) have distinct features at $\omega=\omega_{l}^{*}$ as well as at a certain frequency $\omega_{c}$ lying below $\omega_{\tau}^{*}$. This frequency corresponds to the crossing of a longitudinal acoustic mode, polarized along the $c$ axis, with a transversely polarized optical mode propagating in the same direction. The velocity of sound in this acoustic mode is $s_{l}^{(c)} \sim \sqrt{C_{33}}$ (in the present model the elastic moduli $C_{i k}$ satisfy the relations $C_{11}=2.125 C_{33}=3 C_{66}$ 

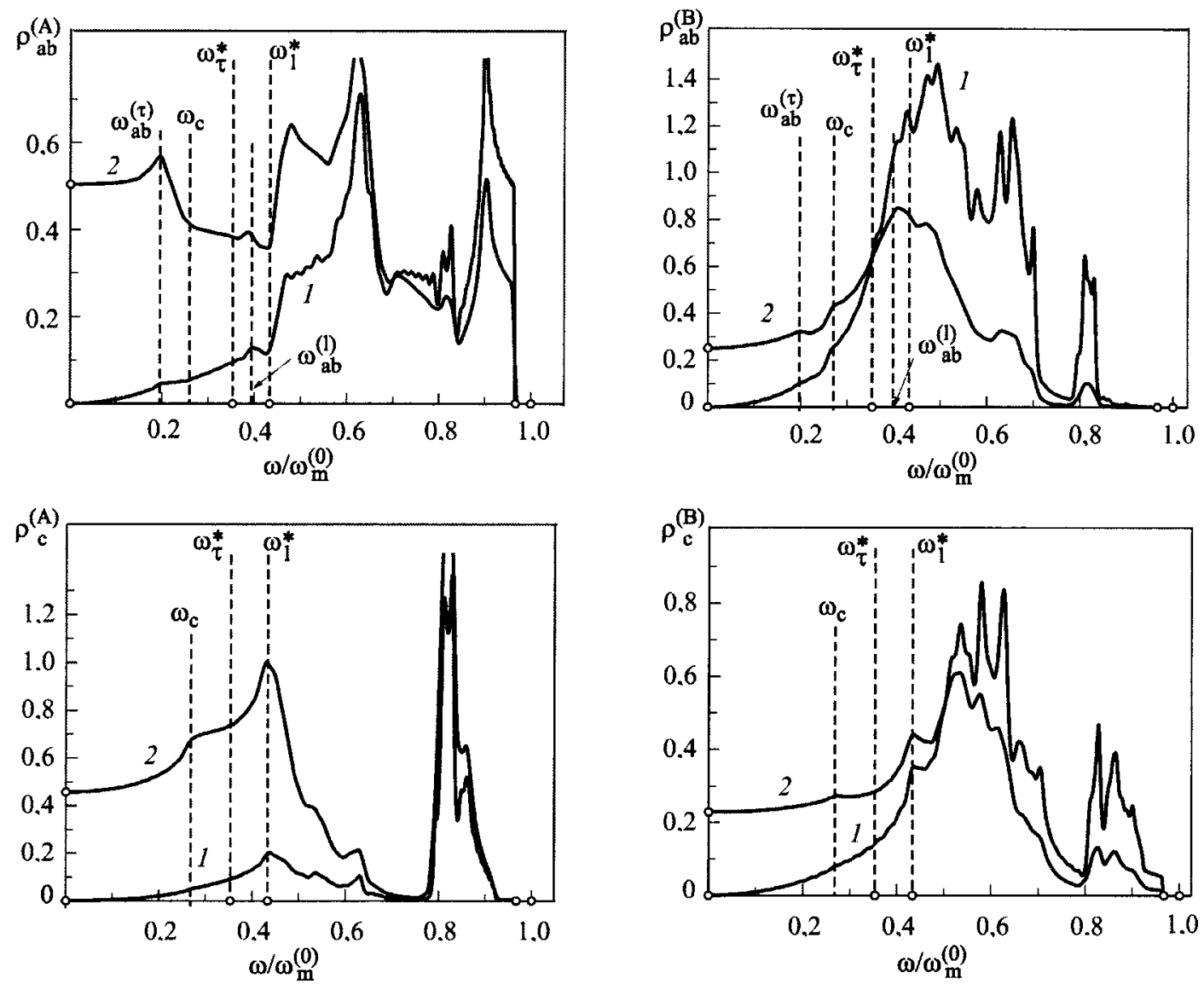

FIG. 7. Spectral densities (curves 1) and their ratio to the squared frequency (curves 2), corresponding to displacements of atoms of different sublattices of the experimental structure along different crystallographic directions.

$\left.=7.5 C_{44}\right)$. The maximum frequency of the optical mode equals $\omega_{\tau}^{*}$.

The spectral densities $\rho_{a b}^{(A)}(\omega)$ and $\rho_{a b}^{(B)}(\omega)$ have additional features at frequencies $\omega_{a b}^{(\tau)}<\omega_{\tau}^{*}$ and $\omega_{a b}^{(l)} \in\left(\omega_{\tau}^{*}, \omega_{l}^{*}\right)$. These features are related with the crossing of the acoustic branches with a low-frequency optical mode which is polarized along the $c$ axis. Three acoustic waves differing substantially from one another propagate in the basal plane-a longitudinal wave $\left(s_{l} \sim \sqrt{C_{11}}\right)$ and two transverse waves polarized in the basal plane $\left(s_{\tau} \sim \sqrt{C_{66}}=s_{l} / \sqrt{3}\right)$ and polarized along the $c$ axis $\left(s_{n} \sim \sqrt{C_{44}}=s_{l} / \sqrt{7.5}\right)$. The first two acoustic modes cross the low-frequency optical mode. In this optical mode the frequency of the vibrations at $k=0$ equals $\omega_{l}^{*}$, and at the boundary of the first Brillouin zone (at the point $K$ ) it joins the slowest acoustic mode, polarized along the $c$ axis. Thus this mode possesses appreciable dispersion, which gives rise to the smallness of the value of $\omega_{a b}^{(\tau)}$ (the frequency $\omega_{a b}^{(\tau)}<\omega_{c}$, since $\left.s_{\tau}<s_{l}^{(c)}\right)$ and the smearing of the feature near $\omega_{a b}^{(l)}$.

We note that the crossing of the low-frequency optical modes with acoustic modes is a well-established fact for
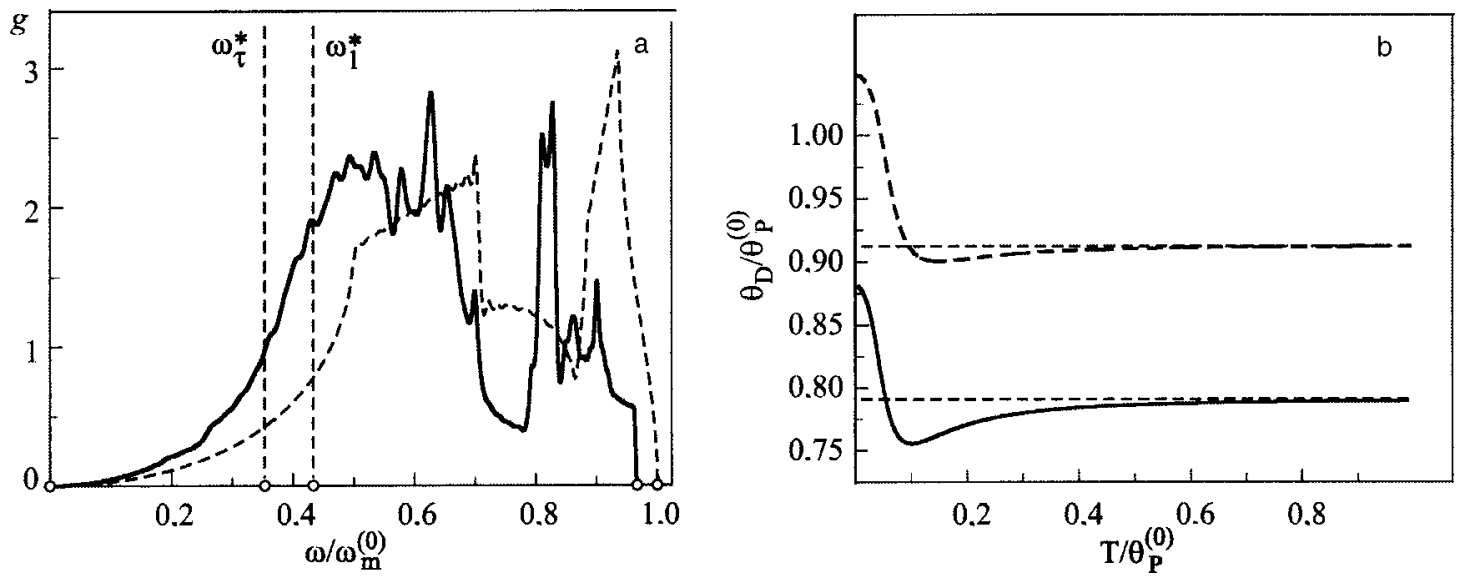

FIG. 8. Phonon density of states (a) and temperature dependence of $\Theta_{D}$ (b) of a layered crystal with a three-atom unit cell (solid curves) in comparison with the analogous characteristics of an ideal fcc lattice with a central interaction of the nearest neighbors (dashed curves). 
many layered crystals with polyatomic unit cells (see, for example, Refs. 51 and 52).

As Fig. 7 (curves 1 and 2) clearly shows, all spectral densities in a quite wide region of low frequencies $\propto \omega^{2}$, i.e. ordinary three-dimensional behavior should be characteristic for the phonon spectrum and the low-temperature thermodynamic quantities determined by it. Consequently, the lowfrequency features in the phonon density of states can be described in terms of the deviations of the density of states from $g_{D}^{(3)}(\omega)$, specifically, as the temperature dependence of the Debye temperature which can be calculated from the relations (2) and (3).

Figure 8 shows the phonon density of states (7) (a) and the temperature dependence of $\Theta_{D}$ for the model considered here (b) and, for comparison, the analogous characteristics of the "initial" fcc lattice (lattice of type $A$ atoms). As a result of the weakening (as compared with the $A$ lattice) of some force bonds, the phonon density of states increases at low frequencies and, therefore, the Debye temperature decreases. The presence of features which are associated with the retardation of fast acoustic phonons as a result of their scattering by slow optical phonons gives rise to the formation of a distinct low-temperature minimum in the function $\Theta_{D}$.

In summary, scattering of fast acoustic phonons by slow optical phonons can occur in crystalline ordered heterogeneous structures. This scattering, similar to the scattering of such phonons by quasilocalized vibrations and disordered systems, results in the formation of similar low-frequency features in the phonon densities of states, which are manifested similarly in the behavior of the low-temperature vibrational characteristics. These features can be identified with Ioffe-Regel' crossover because of the way they arise and manifest themselves. We note that these features are completely analogous to those studied in Refs. 39 and 40.

\section{CONCLUSIONS}

It can be concluded on the basis of the results obtained in the preceding sections that the nature of the Ioffe-Regel' features and disordered systems is similar to the nature of the formation of the first van ove Hove Hove singularity in structures with crystalline regularity in the arrangement of the atoms.

The Ioffe-Regel' feature, defined as the boundary between rapidly propagating phonons ("propagons") and slower phonons ("diffusons"), ${ }^{40}$ corresponds to the wavelength of rapidly propagating phonons for which these phonons start to feel the defect structure.

The frequency $\omega^{*}$ of the first van Hove singularity corresponds to the wavelength $\lambda$ of fast acoustic phonons starting at which (i.e. for $\lambda<\lambda^{*}$ ) the propagation of these phonons changes qualitatively - the discreteness of the crystal lattice starts to affect the propagation. The average velocity and the average wavelength of acoustic phonons change abruptly. Consequently, the first van Hove singularity also separates fast acoustic phonons with a nearly acoustic dispersion law (the same "propagons") from much slower phonons ("diffusons") whose dispersion law is different from the acoustic dispersion law.

The presence of different kinds of defects in a crystal can result in additional retardation of fast acoustic phonons, which under certain conditions can also be manifested as a jump in the frequency dependence of their group velocity. It is shown in Sec. II above that these conditions reduce to the formation of quasilocalized states in the phonon spectrum, i.e. very slowly propagating phonons localized near any defect. These states can not only give rise to low-frequency maxima in the "propagon" frequency range of the initial idea of crystal but they can also serve as scattering centers for fast acoustic phonons corresponding to the vibrations of atoms of the main lattice. It was shown that defect clusters which are at least two interatomic distances in size can serve as effective scattering centers. Consequently, the parameter $l>2 a_{0}$, which can be taken as the disorder parameter, becomes comparable to the wavelength (Ioffe-Regel' limit ${ }^{39,40}$ ). It is only in this case that quasilocalized states with frequencies below the frequency of the first van Hove singularity $\omega^{*}$ can form, and it is only in this case that phonons with frequencies $\omega$ $<\omega^{*}$ ("propagons") will be effectively scattered by these quasilocal vibrations. Such an abrupt decrease of the group velocity of phonons shifts the boundary separating "propagons" and "diffusons" to lower frequencies (to the frequency of quasilocalized vibrations $\left.\omega_{q l}<\omega^{*}\right)$, and the frequency $\omega_{q l}$ is the frequency of the Ioffe-Regel' feature for the given system. It was shown in Sec. II above that the formation of a quasilocalized vibration with the frequency $\omega_{q l}$ may not be accompanied by the appearance of a lowfrequency peak in the phonon density of states.

The Ioffe-Regel' feature and the first Van Hove singularity manifest in the same way-as a kink-in the phonon density of states of three-dimensional crystal lattices. The same features arise in the density of states of layered crystals with a complex lattice and of other heterogeneous structures with crystalline order as a result of the presence of lowfrequency optical modes (phonons with a low group velocity) in their phonon spectra. As shown in Sec. III above, the nature of such features is still the same-scattering of fast acoustic phonons by slow phonons.

The phonon enrichment of the low-frequency region of a quasicontinuous spectrum causes the density of states to deviate appreciably from the Debye form. It was shown above that this deviation, often manifested as peaks in the ratio of the phonon density of states to the squared frequency (socalled "boson peaks" or "boson maxima"), is due to the additional dispersion of sound waves. Nonetheless, the lowtemperature specific heat and the temperature dependence, expressed through it, of the Debye temperature are informative characteristics of this redistribution of phonons over the spectrum.

In summary, the present work has clarified at the microscopic level the reasons for the appearance of characteristic differences of the phonon spectrum of solids from the Debye spectrum in the low-frequency region. The factors attributable to the appearance of a so-called boson peak and to Ioffe-Regel' crossover were analyzed.

Using the proposed analysis we are continuing A. S. Kosevich's investigations, ${ }^{39,40}$ which he performed while studying the lattice dynamics of disordered solid systems. We were fortunate to work side by side with Arnol'd Markovich Kosevich - a prominent scientist and exceptionally kind and charming human being-for many years. The range of his 
scientific interests was unusually wide, and in each sphere of his multifaceted scientific work he left a truly distinct mark. There remain not only results which are now in textbooks and not only methods which he developed and which have enriched the arsenal of modern theoretical physics but also ideas which he did not have the time to develop completely.

In the present work we have tried to realize one such idea in part-the manifestation of scattering of plane waves by spherical waves in the vibrational characteristics of real crystals. We dedicate this work to the blessed memory of Arnol'd Markovich. This work was supported by grants No. 23/07-N from the National Academy of Sciences of Ukraine and No. 4119 from the Ukrainian Center for Science and Technology.

${ }^{a)}$ E-mail: gospodarev@ilt.kharkov.ua

${ }^{1}$ I. M. Lifshits, Dokl. Akad. Nauk SSSR 48, 83 (1945).

${ }^{2}$ I. M. Lifshits, Usp. Mat. Nauk 7, 171 (1952).

${ }^{3}$ I. M. Lifshitz and A. M. Kosevich, Rep. Prog. Phys. 29, 217 (1966).

${ }^{4}$ I. M. Lifshitz, S. A. Gredeskul, and L. A. Pastur, Introduction to the Theory of Disordered Systems, Wiley, New York (1988).

${ }^{5}$ A. M. Kosevich, Principles of Crystal Lattice Dynamics, Nauka, Moscow (1972).

${ }^{6}$ A. M. Kosevich, Physics of Real Crystals, Naukova dumka, Kiev (1981).

${ }^{7}$ A. M. Kossevich, The Crystal Lattice: Phonons, Solitons, Dislocations, Wiley-VCH, Berlin (1999).

${ }^{8}$ Amorphous Solid-Low Temperature Properties, edited by W. A. Phillips, Springer, Berlin (1981).

${ }^{9}$ N. Ahmad, K. W. Hutt, and W. A. Phillips, J. Phys. C 19, 3765 (1986).

${ }^{10}$ D. Engberg, A. Wiscnewski, V. Buchenau, L. Borjesson, A. J. Dianoux, A. D. Sokolov, and L. M. Torell, Phys. Rev. B 59, 4053 (1999).

${ }^{11}$ A. Matic, D. Endberg, C. Masciovecchio, and L. Borjesson, Phys. Rev. Lett. 86, 3803 (2001).

${ }^{12}$ C. A. Angel, K. L. Nqai, G. B. McKenna, P. F. McMillan, and S. W. Martin, J. Appl. Phys. 88, 3113 (2000).

${ }^{13}$ M. Forett, R. Vacher, E. Courtens, and G. Monaco, Phys. Rev. B 66, 024204 (2002).

${ }^{14}$ T. Scopigno, E. Pontecorvo, R. Di Leonardo, M. Krisch, G. Monaco, G. Ruocco, B. Ruzichka, and F. Sette, J. Phys.: Condens. Matter 15, S1269 (2003).

${ }^{15}$ A. I. Chumakov, I. Sergeev, U. van Burck, W. Schrimacher, T. Asthalter, R. Ruffer, O. Leupold, and W. Petry, Phys. Rev. Lett. 92, 245508 (2004).

${ }^{16}$ V. G. Karpov, V. G. Klinger, and F. N. Ignat'ev, Sov. Phys. JETP 57, 439 (1983).

${ }^{17}$ V. L. Gurevich, D. A. Parshin, J. Pelous, and H. R. Schrober, Phys. Rev. B 48, 16318 (1993).

${ }^{18}$ V. L. Gurevich, D. A. Parshin, and H. R. Schrober, Phys. Rev. B 67 , 094203 (2003)

${ }^{19}$ U. Buchenau, Yu. M. Galperin, V. L. Gurevich, D. A. Parshin, M. A. Ramos, and H. R. Schrober, Phys. Rev. B 46, 2798 (1992).

${ }^{20}$ W. Schrimacher and M. Wagener, Solid State Commun. 86, 597 (1993).
${ }^{21}$ R. Kunh and U. Horstrann, Phys. Rev. Lett. 78, 4067 (1997).

${ }^{22}$ W. Schrimacher and M. Wagener, Phys. Rev. Lett. 81, 136 (1998).

${ }^{23}$ W. Gotze and M. R. Mayr, Phys. Rev. E 61, 587 (2000).

${ }^{24}$ S. N. Taraskin, Y. L. Loh, G. Natarajan, and S. R. Elliott, Phys. Rev. Lett. 86, 1225 (2001)

${ }^{25}$ J. W. Kantelhardt, S. Russ, and A. Bunde, Phys. Rev. B 63, 064302 (2001).

${ }^{26}$ E. Maurer and W. Schrimacher, J. Low Temp. Phys. 137, 453 (2004).

${ }^{27}$ T. S. Grigera, V. Martin-Mayor, G. Parisi, and P. Verrochio, Nature (London) 422, 289 (2003)

${ }^{28}$ M. Turlakov, Phys. Rev. Lett. 93, 035501 (2004).

${ }^{29}$ V. Gurarie and A. Altland, Phys. Rev. Lett. 94, 245502 (2004).

${ }^{30}$ J. L. Feldman, P. B. Allen, and S. H. Bickman, Phys. Rev. B 59, 3551 (1999).

${ }^{31}$ S. N. Taraskin and S. R. Elliott, Phys. Rev. B 61, 12017 (2000).

${ }^{32}$ S. I. Simdyankin, S. N. Taraskin, M. Elenius, S. R. Elliott, and M. Dzugutov, Phys. Rev. B 65, 104302 (2002).

${ }^{33}$ R. Dell'Anna, G. Russo, M. Sampoli, and G. Viliani, Phys. Rev. Lett. 80, 1236 (1998).

${ }^{34}$ M. C. C. Ribeiro, M. Wilson, and P. A. Madden, J. Chem. Phys. 108, 9027 (1998).

${ }^{35}$ P. Jund and R. Jullien, Phys. Rev. B 59, 13707 (1999).

${ }^{36}$ J. Horbach et al., Eur. Phys. J. B 19, 531 (2001).

${ }^{37}$ P. B. Allen, J. L. Feldman, J. Fabian, and F. Wooten, Philos. Mag. B 72, 1715 (1999).

${ }^{38}$ S. R. Elliott, Europhys. Lett. 19, 201 (1992).

${ }^{39}$ M. I. Klinger and A. M. Kosevich, Phys. Lett. A 280, 365 (2001).

${ }^{40}$ M. I. Klinger and A. M. Kosevich, Phys. Lett. A 295, 311 (2002).

${ }^{41}$ A. Feher, I. M. Yurkin, L. I. Deich, M. Orendach, and I. D. Turyanitsa, Physica B 194-196, 395 (1994).

${ }^{42}$ S. A. Alexander and R. Orbach, J. Plasma Phys. 43, 625 (1982).

${ }^{43}$ V. K. Malinovsky, A. P. Sokolov, and V. N. Novikov, Phys. Lett. A 153, 63 (1991).

${ }^{44}$ W. Steurer, A. Apfolter, M. Koch, W. E. Ernst, B. Holst, E. Sondergard, and J. R. Manson, Phys. Rev. Lett. 99, 035503 (2007).

${ }^{45}$ U. Buchenau, A. Wisschnewski, M. Ohl, and E. Fabiani, arXiv:condmat10407136, v.1, 6 Julay (2004).

${ }^{46}$ V. A. Konstantinov, V. G. Manzhelii, V. P. Revyakin, V. V. Sagan, and O. I. Pursky, J. Phys.: Condens. Matter 18, 901 (2006).

${ }^{47} \mathrm{H}$. Liebfried, Microscopic Theory of the Mechanical and Thermal Properties of Crystals, Fizmatgiz, Moscow (1963).

${ }^{48}$ L. D. Landau and E. M. Lifshitz, Statistical Physics, Nauka, Moscow (1964).

${ }^{49}$ M. I. Bagatskii, S. B. Feodosyev, I. A. Gospodarev, O. V. Kotlyar, E. V. Manzhelii, A. V. Nedzvetski, and E. S. Syrkin, Fiz. Nizk. Temp. 33, 741 (2007) [Low Temp. Phys. 33, 564 (2007)].

${ }^{50}$ M. I. Bagatskiĭ, E. S. Syrkin, and S. B. Feodos'ev, Fiz. Nizk. Temp. 18, 629 (1992) [Low Temp. Phys. 18, 445 (1992)].

${ }^{51}$ N. Wakabayashi, H. G. Smith, and R. Shanks, Phys. Lett. A 50, 367 (1974).

${ }^{52}$ D. E. Moncton, J. D. Axe, and F. J. Di Salvo, Phys. Rev. B 16, 801 (1977).

${ }^{53}$ E. S. Syrkin and S. B. Feodos'ev, Fiz. Nizk. Temp. 8 (1982) [Low Temp. Phys. 8 (1962)].

${ }^{54}$ V. V. Tarasov, Zh. Fiz. Khim. 24, 111 (1950).

${ }^{55}$ I. M. Lifzhits, Zh. Eksp. Teor. Fiz. 22, 475 (1952) [SIAM J. Control Optim.].

Translated by M. E. Alferieff 